Scientiæ studia, São Paulo, v. 8, n. 2, p. 273-91, 2010

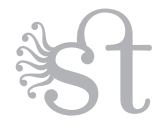

\title{
Medición cuántica y decoherencia: ¿qué medimos cuando medimos?
}

\author{
Olimpia Lombardi \& Leonardo VAnni
}

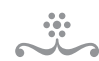

RESUMEN

El problema de la base privilegiada consiste en dar cuenta de la base que define el observable medido en una medición cuántica, dada la supuesta ambigüedad teórica en la definición de dicho observable. El propósito del presente artículo es analizar este problema escasamente explorado. El objetivo final es argumentar que, contrariamente a la opinión más difundida, la teoría de la decoherencia no aporta una solución a dicho problema, no debido a su propia incapacidad, sino porque, en realidad, se trata de un pseudo-problema que no requiere solución alguna.

Palabras-clave $\bullet$ Aparato de medición. Entorno. Base del espacio de Hilbert. Superposición.

\section{INTRODUCGIÓN}

Entre los múltiples desafíos que presenta la mecánica cuántica, el problema de la medición es sin duda el más discutido, tal vez porque es el que enfrenta el cuerpo teórico con la evidencia empírica más directa. Resumidamente, dicho problema puede expresarse en los siguientes términos: ¿cómo es posible explicar, en una medición cuántica, el valor definido de los observables del aparato macroscópico, cuando desde el punto de vista cuántico el sistema se encuentra en una superposición de estados? Cataratas de tinta han corrido tras el intento de encontrar una respuesta adecuada a las dificultades que plantea esta pregunta, e incluso muchas interpretaciones han sido diseñadas específicamente para suministrar una solución conceptualmente admisible al problema. En efecto, puede discutirse acerca de la naturaleza de las partículas elementales, del modo en que interactúan a través del espacio o de la interpretación de la estadística que generan. Pero no podemos negar que, aun cuando una partícula cuántica se encuentre en un estado de superposición, al efectuar una medición sobre ella observamos la aguja del dispositivo de medición en una posición definida.

La respuesta tradicional al problema de la medición se basa en la llamada 'hipótesis del colapso', formulada por primera vez por Werner Heisenberg en su famoso artículo de 1927 en términos de "reducción del paquete de onda" (cf. Heisenberg, 1927). 
Según esta hipótesis, los sistemas cuánticos desarrollan dos tipos de evolución temporal: una evolución determinista y unitaria cuando no son observados, y una transición indeterminista y no-unitaria (el "colapso" de la función de onda) al ser medidos. Dicha transición conduciría al sistema de su estado de superposición original a otro estado en el cual sistema y aparato de medición adquieren propiedades definidas.

Actualmente, en el ámbito de la física el problema de la medición se aborda a partir de la teoría de la decoherencia inducida por el entorno (environment-induced decoherence). Este programa teórico, desarrollado por un grupo liderado por Wojciech Zurek (1981, 1982, 1991, 2003) y actualmente con sede en el laboratorio de Los Alamos, se basa en el estudio de los efectos de la interacción entre un sistema cuántico, considerado como un sistema abierto, y su entorno. De acuerdo con esa teoría, la interacción sistema-entorno conduce al proceso de decoherencia que selecciona las propiedades ("observables") del sistema que adquieren valores definidos.

A su vez, con la difusión de la teoría de la decoherencia, incluso el propio problema de la medición se ha reformulado, ampliando sus alcances. Tal como indica Schlosshauer (2004, p. 1270), actualmente las dificultades conceptuales de la medición cuántica pueden concentrarse en torno a dos núcleos:

- El problema de la lectura definida (definite outcome), que consiste en la pregunta tradicional acerca de la medición cuántica: ¿por qué percibimos una lectura definida en el dispositivo de medición cuando su estado es una superposición de lecturas posibles?

- El problema de la base privilegiada (preferred basis): dado que existe una ambigüedad teórica en la definición del observable medido debido a la posibilidad matemática de un cambio de base en la expresión del estado del sistema, ¿qué fenómeno físico selecciona tal observable?

Mucho se ha discutido acerca de la capacidad de la teoría de la decoherencia para resolver el problema tradicional de la medición, esto es, el problema de la lectura definida. No obstante la amplia adhesión que despierta el programa, diversas voces se han alzado para alertar contra la confianza excesiva en el papel de la decoherencia para suministrar una respuesta al primer problema. Por el contrario, el problema de la base privilegiada ha sido mucho menos discutido bajo el supuesto de que su solución es uno de los méritos más evidentes de la teoría de la decoherencia. En efecto, incluso muchos, de quienes impugnan la capacidad de la teoría para resolver el problema de la lectura definida, admiten que la decoherencia es el proceso físico que selecciona unívocamente el observable a medir. 
El propósito del presente artículo no consiste en evaluar la respuesta que la teoría de la decoherencia brinda al problema de la base privilegiada, sino que enfrenta una tarea lógicamente previa, o sea, el análisis teórico-conceptual del problema mismo, tal como ha sido formulado y recogido en la bibliografía sobre el tema. El objetivo final consiste en argumentar que, cuando la medición se concibe no exclusivamente en términos del estado correlacionado final, sino como un proceso que brinda información acerca del sistema a medir, el problema de la base privilegiada se disuelve como tal. Sobre la base del estudio de los elementos que intervienen en dicho proceso, se intentará mostrar que la formulación del problema es el resultado de una reconstrucción incompleta de la medición cuántica, que desemboca en conclusiones reñidas tanto con la teoría como con la práctica experimental efectiva.

\section{LA MEDIGIÓN GUÁNTICA}

En la formulación tradicional debida a John von Neumann, la medición cuántica es concebida como una interacción entre el sistema a medir $S$ y un sistema $M$, el aparato de medición. El sistema $S$, representado en el espacio de Hilbert $\mathrm{H}_{S}$, posee un observable $A=\sum_{i} a_{i}\left|a_{i}\right\rangle\left\langle a_{i}\right|$, donde los $a_{i}$ son autovalores de $A$ y $\left\{\left|a_{i}\right\rangle\right\}$ es una base de $\mathrm{H}_{S}$. El aparato $M$, representado en el espacio de Hilbert $\mathrm{H}_{M}$, posee un observable $P=\sum p_{i}\left|p_{i}\right\rangle\left\langle p_{i}\right|$, donde los $p_{i}$ son autovalores de $P$ y $\left\{\left|p_{i}\right\rangle\right\}$ es una base de $\mathrm{H}_{M}$. A fin de cumplir el papel de "aguja" o "puntero" ("pointer") del aparato, $P$ debe poseer autovalores diferentes y macroscópicamente distinguibles. Supóngase que, antes de la interacción, $S$ se encuentra en una superposición de los autovectores del observable $A$, y $M$ se encuentra en un estado base $\left|p_{0}\right\rangle$ ("ready-to-measure state") que puede ser definido como el cero de la aguja. Inicialmente el sistema compuesto $S+M$ se encontrará en un estado $\left|\Psi_{0}\right\rangle \in \mathrm{H}_{S} \otimes \mathrm{H}_{M}$ :

$$
\left|\Psi_{0}\right\rangle=\sum_{i} c_{i}\left|a_{i}\right\rangle \otimes\left|p_{0}\right\rangle
$$

A través de la interacción, el sistema compuesto evolucionará, de acuerdo con la ecuación de Schrödinger, hasta un nuevo estado $\left|\Psi_{f}\right\rangle$, donde los autovectores del observable $A$ en el sistema $S$ quedan correlacionados con los autovectores del observable $P$ en el aparato $M$ :

$$
\left|\Psi_{0}\right\rangle=\sum_{i} c_{i}\left|a_{i}\right\rangle \otimes\left|p_{0}\right\rangle \longrightarrow\left|\Psi_{f}\right\rangle=\sum_{i} c_{i}\left|a_{i}\right\rangle \otimes\left|p_{i}\right\rangle
$$


Sin embargo, a pesar de la perfecta correlación, la dificultad reside en la naturaleza del estado final: $\left|\Psi_{f}\right\rangle$ es una superposición de estados $\left|a_{i}\right\rangle \otimes\left|p_{i}\right\rangle$; por lo tanto, sin un supuesto interpretativo adicional se carece de argumentos para dar cuenta del hecho de que el puntero $P$ adquiera uno de sus autovalores $p_{i}$ de manera definida.

Históricamente, la hipótesis del colapso fue la primera respuesta al problema de la lectura definida. Según esta hipótesis, en el instante de la medición, el sistema compuesto $S+M$ efectúa una transición indeterminista y no unitaria (el "colapso") que convierte el estado $\left|\Psi_{f}\right\rangle$ en uno de los estados de la superposición, digamos, $\left|\varphi_{k}\right\rangle$ :

$$
\left|\Psi_{f}\right\rangle=\sum_{i} c_{i}\left|a_{i}\right\rangle \otimes\left|p_{i}\right\rangle=\sum_{i} c_{i}\left|\varphi_{i}\right\rangle \longrightarrow\left|\varphi_{k}\right\rangle=\left|a_{k}\right\rangle \otimes\left|p_{k}\right\rangle
$$

Puesto que $\left|\varphi_{k}\right\rangle$ ya no es una superposición, se infiere que el observable $A$ adquiere el valor $a_{k}$ y el observable $P$ adquiere el valor $p_{k}$. Dado que el colapso es un proceso indeterminista, la probabilidad de que $\left|\Psi_{f}\right\rangle$ "colapse" en cada estado $\left|\varphi_{i}\right\rangle$ es $\left|c_{i}\right|^{2}$. Esto significa que, si se efectúan muchas mediciones particulares sobre el mismo sistema en las mismas condiciones iniciales, se obtiene cada estado $\left|\varphi_{i}\right\rangle$ con una frecuencia que se aproximará a $\left|c_{i}\right|^{2}$ a medida que se aumente el número de mediciones. Por lo tanto, si se define un ensemble de mediciones a la manera de la mecánica estadística (cf. Ballentine, 1998), el estado del ensemble luego del colapso podrá representarse mediante un operador densidad $\rho_{m}$ :

$$
\rho_{m}=\sum_{i}\left|c_{i}\right|^{2}\left|a_{i}\right\rangle \otimes\left|p_{i}\right\rangle\left\langle a_{i}\right| \otimes\left\langle p_{i}\right|
$$

Siempre según la hipótesis del colapso, el operador densidad $\rho m$ representa un estado mezcla susceptible de una interpretación por ignorancia, esto es, puede ser interpretado como expresando que el sistema compuesto se encuentra efectivamente en alguno de los estados $\left|a_{i}\right\rangle \otimes\left|p_{i}\right\rangle$, pero el observador desconoce en qué estado particular, y sólo puede conocer la probabilidad $\left|c_{i}\right|^{2}$ correspondiente a cada uno de ellos. De este modo, si efectivamente ocurre $\left|a_{\alpha}\right\rangle \otimes\left|p_{\alpha}\right\rangle$, podríamos afirmar que el observable $P$, puntero del aparato, adquiere un valor definido $p_{\alpha}$, y esto indicaría que el sistema $S$ se encuentra en el estado $\left|a_{\alpha}\right\rangle$.

Si bien la hipótesis del colapso es una respuesta "natural" al problema de la lectura definida, a través de los años fue demostrando sus flancos débiles (cf. Jammer, 1974; Hughes, 1989; Bub, 1997). La principal objeción dirigida contra esta interpretación de la medición cuántica es la que se refiere al carácter ad hoc de la hipótesis: el colapso sería un proceso físico del cual, sin embargo, no da cuenta la teoría; de este modo queda indefinido el tipo de interacción que daría lugar a dicho proceso, así como el instante preciso en el que ocurriría. Por ello, actualmente la hipótesis del colapso no 
se adopta en su forma original en las discusiones acerca de los fundamentos de la mecánica cuántica. Pero esto ha obligado a los especialistas a buscar nuevas respuestas: muchas interpretaciones han sido diseñadas específicamente para suministrar una solución conceptualmente adecuada al problema. La teoría de la decoherencia, en cambio, se presenta como un enfoque que, desde la propia física teórica, resuelve las dificultades de la medición cuántica.

\section{LA TEORÍA DE LA DEGOHERENGIA}

Como señalan varios autores (Leggett, 1987; Bub, 1997), la teoría de la decoherencia se ha convertido en la "nueva ortodoxia" en la comprensión de la mecánica cuántica. Las raíces del programa de la decoherencia se encuentran en los estudios de sistemas abiertos durante la década del 1970 (cf. Zeh, 1970). Sobre la base de estos trabajos, Zurek y sus colaboradores (cf. Paz \& Zurek, 2002; Zurek, 2003) desarrollaron la idea de que los sistemas macroscópicos, tales como los aparatos de medición, nunca están aislados sino que interactúan significativamente con su entorno. Según Zurek $(1981,1982,1991)$, es esta interacción el proceso que selecciona un pequeño subconjunto de estados en el espacio de Hilbert, precisamente los estados que se manifestarán de un modo clásico, como las posiciones definidas del puntero en el dispositivo de medición.

Consideremos cómo actúa la decoherencia en la medición cuántica (cf. Paz \& Zurek, 2002). Tal como señalamos en la sección anterior, luego de la interacción entre el sistema $S$ y el aparato $M$, el sistema compuesto $S+M$ se encuentra en un estado de superposición:

$$
\left|\Psi_{f}\right\rangle=\sum_{i} c_{i}\left|a_{i}\right\rangle \otimes\left|p_{i}\right\rangle
$$

Por simplicidad, supongamos que el observable $A$ del sistema $S$ a medir tiene sólo dos autoestados, $\left|a_{1}\right\rangle$ y $\left|a_{2}\right\rangle$, de modo tal que el estado $\left|\Psi_{f}\right\rangle$ resulta

$$
\left|\Psi_{f}\right\rangle=c_{1}\left|a_{1}\right\rangle \otimes\left|p_{1}\right\rangle+c_{2}\left|a_{2}\right\rangle \otimes\left|p_{2}\right\rangle
$$

Pero, según la teoría de la decoherencia, este sistema no se encuentra aislado sino en interacción con un entorno $E$ de un enorme número de grados de libertad. Supongamos que $E$ está conformado por un enorme número $N$ de partículas de spin $1 / 2$ que no interactúan entre sí. Por lo tanto, en un instante inicial $t=0$, el estado del sistema compuesto $S+M+E$ resulta 


$$
\begin{array}{r}
\text { Olimpia Lombardi \& Leonardo Vanni } \\
\left|\Psi_{S M E}(t=0)\right\rangle=\left(c_{1}\left|a_{1}\right\rangle \otimes\left|p_{1}\right\rangle+c_{2}\left|a_{2}\right\rangle \otimes\left|p_{2}\right\rangle\right) \otimes \prod_{k=1}^{N}\left(\gamma_{k}|\uparrow\rangle_{k}+\delta_{k}|\downarrow\rangle_{k}\right) \quad \text { (3.3), }
\end{array}
$$

donde $|\uparrow\rangle_{k} \mathrm{y}|\downarrow\rangle_{k}$ son los autoestados de la componente en la dirección $k$ del spin correspondiente a la partícula $k$. Por simplicidad, se considera que los hamiltonianos del aparato y del entorno son nulos y, además, la interacción entre $S$ y $M$ ya ha cesado. Por lo tanto, la evolución del sistema compuesto $S+M+E$ estará regida por el hamiltoniano de interacción $H_{\text {int }}$. Si se supone que

$$
H_{\text {int }}=\left(\left|a_{1}\right\rangle \otimes\left|p_{1}\right\rangle\left\langle a_{1}\left|\otimes\left\langle p_{1}|+| a_{2}\right\rangle \otimes\right| p_{2}\right\rangle\left\langle a_{2}\right| \otimes\left\langle p_{2}\right|\right) \otimes \sum_{k} g_{k}(|\uparrow\rangle\langle\uparrow|+| \downarrow\rangle\langle\downarrow|)_{k}
$$

bajo la influencia de $H_{\text {int }}$ el estado inicial $\left|\Psi_{\text {SME }}(t=0)\right\rangle$ evolucionará como

$$
\left|\Psi_{S M E}(t)\right\rangle=c_{1}\left|a_{1}\right\rangle \otimes\left|p_{1}\right\rangle \otimes\left|\varepsilon_{1}(t)\right\rangle+c_{2}\left|a_{2}\right\rangle \otimes\left|p_{2}\right\rangle \otimes\left|\varepsilon_{2}(t)\right\rangle
$$

donde

$$
\begin{aligned}
& \left|\varepsilon_{1}(t)\right\rangle=\prod_{k}\left(\delta_{k} \exp \left(i g_{k} t\right)|\uparrow\rangle_{k}+\delta_{k} \exp \left(-i g_{k} t\right)|\downarrow\rangle_{k}\right) \\
& \left|\varepsilon_{2}(t)\right\rangle=\prod_{k}\left(\delta_{k} \exp \left(-i g_{k} t\right)|\uparrow\rangle_{k}+\delta_{k} \exp \left(i g_{k} t\right)|\downarrow\rangle_{k}\right)
\end{aligned}
$$

De este modo, el estado del sistema $S+M$ queda "entrelazado" ("entangled") con el estado del entorno $E$. Si recordamos que el operador densidad correspondiente a un estado puro $|\varphi\rangle$ se define como $\rho=|\varphi\rangle\langle\varphi|$ (cf. Pessoa Júnior, 2003), el operador densi$\operatorname{dad} \rho_{S M E}(t)$ correspondiente a este estado entrelazado será

$$
\begin{aligned}
\rho_{S M E}(t)=\left|\Psi_{S M E}(t)\right\rangle\left\langle\Psi_{S M E}(t)\right| & =\left|c_{1}\right|^{2}\left|a_{1}\right\rangle \otimes\left|p_{1}\right\rangle \otimes\left|\varepsilon_{1}(t)\right\rangle\left\langle a_{1}\right| \otimes\left\langle p_{1}\right| \otimes\left\langle\varepsilon_{1}(t)\right|+ \\
& +c_{1} c_{2}^{*}\left|a_{1}\right\rangle \otimes\left|p_{1}\right\rangle \otimes\left|\varepsilon_{1}(t)\right\rangle\left\langle a_{2}\right| \otimes\left\langle p_{2}\right| \otimes\left\langle\varepsilon_{2}(t)\right|+ \\
& +c_{1}^{*} c_{2}\left|a_{2}\right\rangle \otimes\left|p_{2}\right\rangle \otimes\left|\varepsilon_{2}(t)\right\rangle\left\langle a_{1}\right| \otimes\left\langle p_{1}\right| \otimes\left\langle\varepsilon_{1}(t)\right|+ \\
& +\left|c_{2}\right|^{2}\left|a_{2}\right\rangle \otimes\left|p_{2}\right\rangle \otimes\left|\varepsilon_{2}(t)\right\rangle\left\langle a_{2}\right| \otimes\left\langle p_{2}\right| \otimes\left\langle\varepsilon_{2}(t)\right|
\end{aligned}
$$

donde los términos "cruzados" (términos fuera de la diagonal) representan las correlaciones cuánticas que impiden la interpretación de $\rho_{S M E}(t)$ en términos clásicos.

De acuerdo con Zurek, en cada instante $t$ la descripción del sistema $S+M$ viene dada por el operador densidad reducido $\rho_{r}(t)=\operatorname{Tr}_{(E)}\left[\rho_{S M E}(t)\right]=\Sigma\left\langle\varepsilon_{i}(t)\left|\rho_{S M E}(t)\right| \varepsilon_{i}(t)\right\rangle$, que se obtiene "trazando" (eliminando) los grados de libertad del entorno mediante la operación matemática de traza parcial $\operatorname{Tr}_{(E)}$ (cf. Ballentine, 1998). Por lo tanto, 
Medición GUÁntica y degoherencia: ¿QUé medimos GUANdo medimos?

$$
\begin{aligned}
& \rho_{r}(t)=\left|c_{1}\right|^{2}\left|a_{1}\right\rangle \otimes\left|p_{1}\right\rangle\left\langle a_{1}\left|\otimes\left\langle p_{1}\left|+c_{1} c_{2}^{*} r(t)\right| a_{1}\right\rangle \otimes\right| p_{1}\right\rangle\left\langle a_{2}\right| \otimes\left\langle p_{2}\right|+ \\
& +c_{1}^{*} c_{2} r^{*}(t)\left|a_{2}\right\rangle \otimes\left|p_{2}\right\rangle\left\langle a_{1}\left|\otimes\left\langle\left. p_{1}|+| c_{2}\right|^{2} \mid a_{2}\right\rangle \otimes\right| p_{2}\right\rangle\left\langle a_{2}\right| \otimes\left\langle p_{2}\right|
\end{aligned}
$$

donde el factor $r(t)$,

$$
r(t)=\left\langle\varepsilon_{1}(t) \mid \varepsilon_{2}(t)\right\rangle=\prod_{k}\left[\cos \left(2 g_{k} t\right)+i\left(\left|\gamma_{k}\right|^{2}-\left|\delta_{k}\right|^{2}\right) \operatorname{sen}\left(2 g_{k} t\right)\right]
$$

determina en cada instante el valor de los términos fuera de la diagonal. Zurek y sus colaboradores demuestran que, para $N$ suficientemente grande, a medida que el tiempo transcurre los estados $\left|\varepsilon_{1}(t)\right\rangle$ y $\left|\varepsilon_{2}(t)\right\rangle$ rápidamente tienden hacia la ortogonalidad de modo que, en un intervalo muy corto,

$$
r(t)=\left\langle\varepsilon_{1}(t) \mid \varepsilon_{2}(t)\right\rangle \rightarrow 0
$$

Esto significa que, para un "tiempo de decoherencia" extremadamente pequeño, el operador densidad reducido $\rho_{r}(t)$ converge a

$$
\rho_{r}=\left|c_{1}\right|^{2}\left|a_{1}\right\rangle \otimes\left|p_{1}\right\rangle\left\langle a_{1}\left|\otimes\left\langle\left. p_{1}|+| c_{2}\right|^{2} \mid a_{2}\right\rangle \otimes\right| p_{2}\right\rangle\left\langle a_{2}\right| \otimes\left\langle p_{2}\right|
$$

donde los términos fuera de la diagonal han desaparecido. Según Zurek, pr denota un estado mezcla que sólo contiene los términos correspondientes a las correlaciones clásicas y, por lo tanto, puede interpretarse en términos de ignorancia: el sistema compuesto $S+M$ se encuentra en alguno de los estados $\left|a_{1}\right\rangle \otimes\left|p_{1}\right\rangle$ o $\left|a_{2}\right\rangle \otimes\left|p_{2}\right\rangle$, y las probabilidades $\left|c_{1}\right|^{2} \mathrm{y}\left|c_{2}\right|^{2}$ miden nuestra ignorancia acerca del estado definido del sistema. En consecuencia, la decoherencia inducida por el entorno conduciría al mismo estado ○m introducido por la hipótesis del colapso (ecuación (2.4)), pero sin suponer un proceso físico adicional a la evolución unitaria descripta por la ecuación de Schrödinger. Según Zurek, es precisamente la interacción entre $S+M$ y su entorno $E$ el proceso que hace que "la función de onda parezca haber colapsado" (1981, p. 1517).

Algunos autores consideran que, con la teoría de la decoherencia, se ha alcanzado finalmente la respuesta que demandaba el problema de la medición. Por ejemplo, bajo el supuesto de que una teoría física sólo debe dar cuenta de nuestras percepciones sensibles (las "apariencias"), d'Espagnat sostiene que "la decoherencia explica las recién mencionadas apariencias [las del mundo clásico], y éste es su resultado más importante" (2000, p. 136). De un modo más explícito, en su libro sobre fundamentos de mecánica cuántica, Auletta afirma que "la decoherencia es capaz de resolver prácticamente todos los problemas de la medición que han sido discutidos en los capítulos previos" (2000, p. 289). 
Sin embargo, muchos especialistas no comparten este entusiasmo, y se muestran escépticos respecto de la supuesta solución proporcionada por la teoría de la decoherencia. Por ejemplo, Adler (2003) afirma que, aun cuando el operador densidad reducido $\rho r$ carezca de términos cruzados (ecuación (3.11)), ello no nos autoriza a afirmar que lo que se observa al final del proceso de medición es o bien el evento asociado con $\left|a_{1}\right\rangle \otimes\left|p_{1}\right\rangle$, o bien el evento asociado con $\left|a_{2}\right\rangle \otimes\left|p_{2}\right\rangle ;$; sobre esta base el autor concluye: "no creo que ni los detallados cálculos teóricos ni los recientes resultados experimentales muestren que la decoherencia ha resuelto las dificultades asociadas con la medición cuántica" (2003, p. 136). De acuerdo con Bub (1997), las dificultades son aún más serias: el operador densidad reducido pr no sólo es incapaz de dar cuenta de la ocurrencia de sólo un evento asociado con un valor definido del puntero, sino que, en realidad, es inconsistente con dicha ocurrencia. En efecto, la lectura de $\rho r$ en términos de ignorancia equivale a su interpretación como un estado típico de la mecánica estadística clásica, donde las probabilidades pueden ser concebidas como medidas de nuestra ignorancia acerca de un estado subyacente bien definido que determina los valores precisos de los observables del sistema. Pero este supuesto es inconsistente con la interpretación estándar de la mecánica cuántica, en particular, con el vínculo autoestado-autovalor (eigenstate-eigenvalue link), de acuerdo con el cual un observable tiene valor definido si y sólo si el estado del sistema es un autoestado de dicho observable. Puesto que el estado del sistema compuesto no es un autoestado del observable definido por los $\left|a_{i}\right\rangle \otimes\left|p_{i}\right\rangle$, tal observable no posee valor definido. Éstas y otras consideraciones han conducido incluso a algunos físicos, cuyos aportes fueron centrales en el desarrollo del programa de la decoherencia, a manifestar su escepticismo acerca de la pertinencia de la decoherencia como respuesta al tradicional problema de la medición. Por ejemplo, Joos afirma explícitamente: “¿resuelve la decoherencia el problema de la medición? Claramente no” (2000, p. 14).

\section{El PRoblema DE LA BASE PRIVILEgiada}

Mientras continúa el debate acerca del papel que cumple la decoherencia en el problema de la lectura definida (el tradicional problema de la medición), un nuevo problema se ha instalado en las discusiones sobre el tema: el problema de la base privilegiada (cf. Schlosshauer, 2007, p. $5^{3-5}$ ). La dificultad residiría en la ambigüedad teórica en la definición del observable medido, debida a la posibilidad matemática de expresar el estado del sistema en diferentes bases del espacio de Hilbert. Veamos el problema tal como es formulado por el propio Zurek (1981). 
MEdiGión GUÁNTIGA Y DECOHERENGIA: ¿QUÉ MEdIMOS GUANDo MEDIMOS?

Recordemos la formulación de von Neumann del problema de la medición cuántica:

$$
\left|\Psi_{0}\right\rangle=\sum_{i} c_{i}\left|a_{i}\right\rangle \otimes\left|p_{0}\right\rangle \longrightarrow\left|\Psi_{f}\right\rangle=\sum_{i} c_{i}\left|a_{i}\right\rangle \otimes\left|p_{i}\right\rangle
$$

A través de la interacción, los estados $\left|a_{i}\right\rangle$ del sistema $S$ quedan perfectamente correlacionados con los estados $\left|p_{i}\right\rangle$ del aparato de medición $M$. Según Zurek, precisamente por ello, a la pregunta “¿Qué se ha medido sobre el sistema $S$ ?”, estamos tentados de responder: "el observable $A=\sum_{i} a_{i}\left|a_{i}\right\rangle\left\langle a_{i}\right|$, por supuesto" (1981, p. 1516). Sin embargo, prosigue Zurek, en ciertas circunstancias el formalismo de la mecánica cuántica permite expresar el estado final $\left|\Psi_{f}\right\rangle$ en otra base del espacio de Hilbert del sistema compuesto, digamos, $\left\{\left|a_{i}^{\prime}\right\rangle \otimes\left|p_{i}^{\prime}\right\rangle\right\}$ :

$$
\left|\Psi_{f}\right\rangle=\sum_{i} c_{i}\left|a_{i}\right\rangle \otimes\left|p_{i}\right\rangle=\sum_{i} c_{i}^{\prime}\left|a_{i}^{\prime}\right\rangle \otimes\left|p_{i}^{\prime}\right\rangle
$$

¿Cómo sabemos, entonces, si estamos midiendo el observable $A=\sum_{i} a_{i}\left|a_{i}\right\rangle\left\langle a_{i}\right|$ o el observable $A^{\prime}=\sum_{i} a_{i}^{\prime}\left|a_{i}^{\prime}\right\rangle\left\langle a_{i}^{\prime}\right|$ ?

La posibilidad de expresar el mismo estado en términos de autoestados de otro observable introduciría, así, una ambigüedad fundamental: sin algún elemento adicional no podríamos determinar la base privilegiada del aparato, esto es, "en qué mezcla la función de onda colapsa", tal como lo expresa el propio Zurek en el título de su famoso artículo de 1981. Por lo tanto, no sabríamos qué observable se midió y tampoco cuál es el puntero del aparato de medición, esto es, qué "juego" de lecturas se efectivizará en la medición. Este argumento se generaliza al afirmar:

En el mundo real, incluso cuando no conocemos la lectura, sabemos cuáles son las alternativas y podemos actuar confiadamente como si sólo una de ellas hubiera ocurrido [...]. Pero, ¿cómo un observador, que aún no ha observado el detector, puede expresar su ignorancia acerca de la lectura sin contar con la certeza acerca del "menú" de posibilidades? (Zurek, 1991, p. 38).

No es difícil imaginar la respuesta al problema así planteado en el marco del programa de la decoherencia: es precisamente la decoherencia que resulta de la interacción con el entorno la clave que resolvería la ambigüedad en la elección de la base privilegiada. En efecto, como vimos en el apartado anterior, cuando el sistema compuesto $S+M$ interactúa con el entorno $E$, en tiempos extremadamente cortos las correlaciones cuánticas desaparecen y el sistema compuesto adquiere un estado final (ver ecuación (3.11)) 


$$
\rho_{r}=\sum_{i}\left|c_{i}\right|^{2}\left|a_{i}\right\rangle \otimes\left|p_{i}\right\rangle\left\langle a_{i}\right| \otimes\left\langle p_{i}\right|
$$

De este modo, podríamos afirmar que sobre el sistema $S$ se ha medido el observable $A$ mediante el puntero $P$. En otras palabras, la incesante interacción con el entorno sería el elemento adicional que determinaría "en qué mezcla la función de onda parece haber colapsado" (Zurek, 1981, p. 1517) y, con ello, identificaría unívocamente el observable que se ha medido sobre el sistema $S$.

A diferencia del caso del tradicional problema de la medición, respecto del cual la respuesta ofrecida por la teoría de la decoherencia continúa despertando serias reservas, en el caso del problema de la base privilegiada, la solución propuesta por Zurek ha logrado mucho mayor consenso. Por ejemplo, según Schlosshauer el claro mérito del enfoque de la decoherencia inducida por el entorno consiste en que "la base privilegiada no se elige de un modo ad hoc para lograr que nuestras observaciones sean definidas [...]. Por el contrario, la selección está fundada sobre bases físicas e independientes del observador" (2004, p. 1280). Incluso algunos autores que manifiestan una profunda desconfianza respecto del papel de la decoherencia en la solución del tradicional problema de la medición, admiten la fecundidad del enfoque de Zurek y sus colaboradores para identificar la base privilegiada. En este sentido, Elby afirma: "la decoherencia no puede ayudar a las interpretaciones modales, de estados relativos o de muchos mundos a defenderse de críticas metafísicas. El valor de la decoherencia reside en su capacidad de seleccionar una base especial" (1994, p. 364). Es precisamente por este motivo que la decoherencia ha sido invocada para resolver las dificultades particulares de ciertas interpretaciones de la mecánica cuántica. Por ejemplo, Schlosshauer sostiene que "es razonable anticipar que la decoherencia, inmersa en alguna estructura interpretativa adicional, puede conducir a una descripción completa y consistente del mundo clásico a partir de principios mecánico-cuánticos" (2004, p. 1287 ).

Es claro que la decoherencia identifica una base privilegiada a través del recurso de la interacción con el entorno. También es cierto que, con ello, la teoría de la decoherencia podría brindar un elemento importante a ciertas interpretaciones realistas, como la de muchos mundos, cuyo flanco débil es precisamente la carencia de un criterio para definir la base privilegiada (cf. Wallace, 2002, 2003). Pero, ¿esto significa que, en una medición cuántica, es necesario el entorno para determinar aquello que se mide? En otras palabras, ¿es cierto que sólo a través del proceso de decoherencia sabemos qué medimos cuando medimos? 


\section{El ejemplo de la medición tipo Stern-GerLaGh}

En su presentación del problema de la base privilegiada, Zurek (1981) recurre al caso típico de la medición tipo Stern-Gerlach, donde el momento $P_{z}$ en la dirección $z$ juega el papel de puntero $P$ en la medición, y el estado del sistema se expresa en la base del spin $S z$ en la dirección $z$ :

$$
\begin{gathered}
S_{z}|\uparrow\rangle=s_{z \uparrow}|\uparrow\rangle \quad, \quad S_{z}|\downarrow\rangle=s_{z \downarrow}|\downarrow\rangle \\
P_{z}|+\rangle=p_{+}|+\rangle, \quad P_{z}|-\rangle=p_{-}|-\rangle, \quad P_{z}|0\rangle=p_{0}|0\rangle
\end{gathered}
$$

A su vez, Zurek siempre apela al caso particular en el cual el estado inicial $\left|\Psi_{S}\right\rangle$ de $S$ es

$$
\left|\Psi_{S}\right\rangle=1 / \sqrt{2}(|\uparrow\rangle+|\downarrow\rangle)
$$

En este caso, el proceso de interacción entre $S$ y $M$ puede expresarse:

$$
\left|\Psi_{0}\right\rangle=[1 / \sqrt{2}(|\uparrow\rangle+|\downarrow\rangle)] \otimes|0\rangle \longrightarrow\left|\Psi_{f}\right\rangle=1 / \sqrt{2}\left(|\uparrow\rangle \otimes\left|p_{+}\right\rangle+|\downarrow\rangle \otimes\left|p_{-}\right\rangle\right) \quad \text { (5.4). }
$$

Pero el estado entrelazado $\left|\Psi_{f}\right\rangle$ puede expresarse en otra base, por ejemplo como

$$
\left|\Psi_{f}\right\rangle=1 / \sqrt{2}\left(|\uparrow\rangle \otimes\left|p_{+}\right\rangle+|\downarrow\rangle \otimes\left|p_{-}\right\rangle\right)=1 / \sqrt{2}\left(|\rightarrow\rangle \otimes\left|p_{+}^{\prime}\right\rangle+|\leftarrow\rangle \otimes\left|p^{\prime}{ }_{-}\right\rangle\right) \quad \text { (5.5), }
$$

donde $|\rightarrow\rangle$ y $|\leftarrow\rangle$ son los autovectores del spin $S_{x}$ en dirección $x, y\left|p_{+}{ }_{+}\right\rangle$y $\left|p^{\prime}\right\rangle$ son los autovectores de un nuevo puntero $P$ '. Según el problema de la base privilegiada, sin consultar la salida del aparato de medición, el observador no podría saber si el observable medido es $S_{x}$ o $S_{x}$, incluso en este caso en que ambos observables no conmutan.

Presentado de este modo, el ejemplo de la medición tipo Stern-Gerlach parece ser sólo una ilustración concreta del argumento general presentado en la Sección 4: la ecuación (5.5) sería sólo un caso particular del cambio de base genérico

$$
\left|\Psi_{f}\right\rangle=\sum_{i} c_{i}\left|a_{i}\right\rangle \otimes\left|p_{i}\right\rangle=\sum_{i}{c_{i}^{\prime}}_{i}\left|a_{i}^{\prime}\right\rangle \otimes\left|p^{\prime}{ }_{i}\right\rangle
$$

Sin embargo, este ejemplo aparentemente inocente esconde peculiaridades que, cuando son traídas a la luz, ponen de manifiesto las debilidades del argumento general.

En primer lugar, el hecho de que el cambio de base expresado en el caso particular de la ecuación (5.5) sea correcto no implica que el cambio de base genérico expresado en la ecuación (5.6) sea siempre posible de un modo no trivial. En efecto, por el 
teorema de descomposición biortogonal o teorema de Schmidt se sabe que la descomposición de $\left|\Psi_{f}\right\rangle \in \mathrm{H}_{S} \otimes \mathrm{H}_{M}$ en autoestados $\left.\left|a_{i}\right\rangle \otimes p_{i}\right\rangle$, donde $\left\{\left|a_{i}\right\rangle\right\}$ es una base de $\mathrm{H}_{S} \mathrm{y}$ $\left\{\left|p_{i}\right\rangle\right\}$ es una base de $\mathrm{H}_{M}$, es única en el caso no degenerado, esto es, cuando todos los coeficientes $\left|c_{i}\right|$ son diferentes entre sí. Por lo tanto, el cambio de base expresado en la ecuación (5.6) sólo es posible (esto es, será un cambio de base no trivial, con $\left|a_{i}\right\rangle\left|a_{i}{ }_{i}\right\rangle \mathrm{y}$ $\left.\left|p_{i}\right\rangle \neq\left|p_{i}^{\prime}\right\rangle\right)$ en el caso degenerado, es decir, cuando algunos de los coeficientes ci son iguales en valor absoluto. Ésta es precisamente la situación sobre la que Zurek construye su famoso argumento: el caso de una medición Stern-Gerlach en la cual el estado inicial del sistema $S$ a medir es

$$
\left|\Psi_{S}\right\rangle=\frac{1}{\sqrt{2}}|\uparrow\rangle+\frac{1}{\sqrt{2}}|\downarrow\rangle
$$

donde $c_{1}=c_{2}=1 / \sqrt{ } 2$. Es en esta situación donde el supuesto problema de la ambigüedad de la base se manifestaría. Por lo tanto, el problema de la base privilegiada no es tan general como inicialmente podía suponerse; sólo se manifestaría para algunos estados iniciales del sistema $S$, como en el ejemplo especialmente diseñado por Zurek para presentar su argumento.

En segundo lugar, cabe preguntarse qué relevancia tienen para la medición los estados iniciales degenerados del sistema. En general, el estado inicial $\left|\Psi_{S}\right\rangle$ de $S$ se expresa en la base definida por el observable $S_{z}$ como

$$
\left|\Psi_{S}\right\rangle=c_{1}|\uparrow\rangle+c_{2}|\downarrow\rangle
$$

donde $c_{1}$ y $c_{2}$ son números complejos cualesquiera que cumplen la condición de normalización: $\left|c_{1}\right|^{2}+\left|c_{2}\right|^{2}=1$. Es fácil comprobar, entonces, que el espacio de los pares $\left(\left|c_{1}\right|,\left|c_{2}\right|\right)$ que además cumplen la condición $\left|c_{1}\right|=\left|c_{2}\right|$ tiene una dimensión menor que el espacio de los pares $\left(\left|c_{1}\right|,\left|c_{2}\right|\right)$ que sólo cumplen la condición de normalización. Por lo tanto, los estados iniciales degenerados del sistema son casos tan particulares que forman un espacio de medida nula en el espacio de todos los posibles estados iniciales $\left|\Psi_{s}\right\rangle$. En consecuencia, el ejemplo elegido por Zurek para presentar su argumento de la ambigüedad de la base no es un caso representativo de una situación genérica, sino un caso altamente particular que no puede ser utilizado legítimamente para poner de manifiesto un problema supuestamente general.

Finalmente, para evaluar qué tan grave es para la medición cuántica la posibilidad de expresar el estado $\left|\Psi_{f}\right\rangle$ en distintas bases en el caso degenerado, se impone una última pregunta: ¿qué es una medición cuántica? Es cierto que, por ejemplo, en una medición Stern-Gerlach suele hablarse de "la medición del spin” mediante el momen- 
to. Pero este modo de hablar es sólo una analogía con la medición clásica, donde la correlación entre un observable $A$ del sistema a medir y el puntero $P$ del aparato de medición permite conocer el valor de $A$ a través del valor de $P$ : cuando la columna de mercurio del termómetro coincide con la marca " $3 \eta$ ", medimos el valor $3 \eta^{0} \mathrm{C}$ de la temperatura. Pero el físico experimental sabe que el objetivo de una medición cuántica no es "descubrir" el valor del observable $A$ en cada detección singular: bien conocidas son las dificultades teóricas que acarrea la adjudicación de un valor preciso a un observable cuando el estado del sistema no es un autovector de dicho observable. El propósito de una medición cuántica es reconstruir el estado $\left.\Psi_{S}\right\rangle$ en el que se encontraba el sistema $S$ antes de la interacción. Y para ello serán necesarias muchas detecciones, de modo tal que la frecuencia con la que se detecta cada autovalor $p_{i}$ del puntero $P$ brinde el valor $\left|c_{i}\right|^{2}$ correspondiente. ${ }^{\mathbf{1}}$ Por lo tanto, el aparato de medición se diseña para obtener los valores $\left|c_{i}\right|^{2}$ para cualesquiera valores $c_{i}$, esto es, para cualquier $\left|\Psi_{S}\right\rangle$. En otras palabras, es precisamente el estado inicial $\left|\Psi_{S}\right\rangle=\Sigma c_{i}\left|a_{i}\right\rangle$ el elemento en principio desconocido y que se pretende reconstruir a partir de la medición cuántica.

Una vez que se ha comprendido la especificidad de la medición cuántica y se recuerda el resultado del teorema de Schmidt, el argumento de la ambigüedad de la base pierde su plausibilidad original. En efecto, el arreglo experimental se diseña para reconstruir el estado inicial del sistema $S$, sea cual sea ese estado inicial. El hecho de que, para algunos casos particulares de $\left|\Psi_{S}\right\rangle$ (casos degenerados que tienen medida nula en el espacio de todos los posibles estados $\left.\left|\Psi_{s}\right\rangle\right)$ pueda efectuarse el cambio de base no trivial indicado en la ecuación (5.6) no implica ambigüedad alguna. La medición no se diseña para medir un estado particular del sistema $S$, sino que debe ser efectiva para cualquier $\left|\Psi_{S}\right\rangle$. Por lo tanto, el legítimo puntero $P$ queda unívocamente determinado en los casos en que $\left|\Psi_{S}\right\rangle$ no es degenerado, casos donde, por el teorema de Schmidt, las descomposiciones en bases diferentes, tales como las expresadas en la ecuación (5.5), no son posibles.

Cuando el argumento de la ambigüedad de la base se analiza a la luz de estas consideraciones, es fácil comprobar que Zurek "construye" el problema de la base privilegiada ignorando la naturaleza de la medición cuántica y suponiendo que el problema se reduce a elegir una base para un estado cuántico. Pero una medición involucra un arreglo experimental diseñado para reconstruir (al menos parcialmente) el estado inicial del sistema a medir, sea cual sea ese estado, y por tanto existe una única base $\left.\left|a_{i}\right\rangle \otimes p_{i}\right\rangle$ que

\footnotetext{
1 Este objetivo debe poder lograrse incluso en el caso de una medición no-ideal, esto es, una medición en la cual la correlación entre los autovectores del observable $A$ y los autovectores del puntero $P$ no es perfecta, puesto que la medición ideal es una situación teórica que nunca se cumple en la práctica experimental (cf., para una discusión sobre el tema, Lombardi \& Castagnino, 2008; Castagnino \& Lombardi, 2008).
} 
conserva la correlación necesaria para cualesquiera valores $c_{i}$. En consecuencia, el puntero $P$ queda unívocamente definido, y los detectores deberán ser diseñados específicamente para registrar los valores de ese puntero $P$ y no los valores de cualquier otro puntero $P^{\prime}$.

En definitiva, la pregunta ¿cómo sabemos que estamos midiendo el observable $A$ o el observable $A^{\prime}$ ? puede fácilmente responderse: sabemos que medimos el estado del sistema en la base de $A$ mediante el puntero $P$, y no en la base de $A$ ' mediante el puntero $P^{\prime}$, porque sólo la base $\left|a_{i}\right\rangle \otimes\left|p_{i}\right\rangle$ conserva la correlación entre los valores de ambos observables para cualquier estado inicial $\left|\Psi_{S}\right\rangle$ del sistema $S$. En otras palabras, lo que el observador de la medición desconoce antes de la detección es el estado inicial del sistema a medir, y no el puntero cuyos valores debe detectar o el observable del sistema correlacionado con dicho puntero.

\section{LA MEDIGIÓN COMO PROCESO}

El argumento de la ambigüedad de la base privilegiada se presenta como una consecuencia de un resultado matemático: la posibilidad de expresar un vector de un espacio de Hilbert en cualquier base de dicho espacio. Sin embargo, esta presentación del problema ignora que la medición cuántica es un proceso que se origina en el estado inicial del sistema a medir y, en tanto proceso, debe estar regido por el postulado dinámico de la mecánica cuántica, esto es, la ecuación de Schrödinger. Veamos, entonces, qué conclusiones pueden extraerse del argumento de la ambigüedad de la base cuando la medición se concibe como un proceso cuántico.

Recordemos que, según el modelo de von Neumann, el sistema compuesto $S+M$ se encuentra inicialmente en un estado $\left|\Psi_{0}\right\rangle$. A través de la interacción, $\left|\Psi_{0}\right\rangle$ evoluciona hasta un nuevo estado $\left|\Psi_{f}\right\rangle$ donde los autovectores del observable $A$ en el sistema $S$ quedan correlacionados con los autovectores del observable $P$ en el aparato $M$. De acuerdo con la ecuación de Schrödinger, tal evolución se encuentra determinada por un operador de evolución unitario $U_{t}$ :

$$
\left|\Psi_{0}\right\rangle=\sum_{i} c_{i}\left|a_{i}\right\rangle \otimes\left|p_{0}\right\rangle \longrightarrow\left|\Psi_{f}\right\rangle=U_{t}\left|\Psi_{0}\right\rangle=\sum_{i} c_{i}\left|a_{i}\right\rangle \otimes\left|p_{i}\right\rangle
$$

Según el argumento de la ambigüedad de la base, en este caso decimos que medimos el observable mediante el puntero $P$. Sin embargo, prosigue el argumento, dado que $\left|\Psi_{f}\right\rangle$ puede también expresarse en la base $\left\{\left|a_{i}^{\prime}\right\rangle \otimes\left|p_{i}^{\prime}\right\rangle\right\}$, podríamos igualmente decir que hemos medido el observable $A^{\prime}$ mediante el puntero $P^{\prime}$. 
Para analizar el argumento desde la perspectiva de la medición como proceso, veamos cómo se expresaría la medición de $A^{\prime}$ mediante $P^{\prime}$ :

$$
\left|\Psi_{0}\right\rangle=\sum_{i} c_{i}^{\prime}\left|a_{i}^{\prime}\right\rangle \otimes\left|p_{0}\right\rangle \longrightarrow\left|\Psi_{f}^{\prime}\right\rangle=U_{t}^{\prime}\left|\Psi_{0}^{\prime}\right\rangle=\sum_{i}{c^{\prime}}_{i}\left|a_{i}^{\prime}\right\rangle \otimes\left|p_{i}^{\prime}\right\rangle
$$

Ahora bien, sabemos que $\left|\Psi_{f}\right\rangle=\left|\Psi_{f}^{\prime}\right\rangle$, puesto que se trata del mismo vector expresado en diferentes bases. Por lo tanto,

$$
U_{t}\left|\Psi_{0}\right\rangle=U^{\prime}{ }_{t}\left|\Psi_{0}^{\prime}\right\rangle
$$

Esta última igualdad pone de manifiesto que, en general, las expresiones (6.1) y (6.2) describen mediciones diferentes ya que, si bien conducen a un mismo estado final $\left|\Psi_{f}\right\rangle=\left|\Psi_{f}^{\prime}\right\rangle$, parten de estados iniciales $\left|\Psi_{0}\right\rangle y_{y}\left|\Psi_{0}^{\prime}\right\rangle$ que evolucionan según operadores de evolución $U_{t}$ y $U_{t}^{\prime}$ diferentes.

La pregunta es: ¿podrían las expresiones (6.1) y (6.2), en algún caso, describir la misma medición expresada en bases diferentes? Para responder a esta pregunta, razonemos por el absurdo. El hecho de que las expresiones (6.1) y (6.2) describieran la misma medición en bases diferentes significaría que:

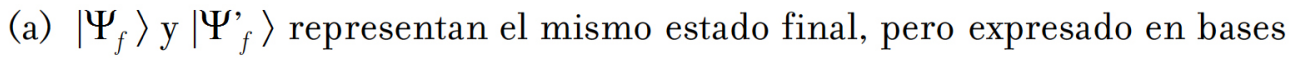
diferentes.

(b) $\left|\Psi_{0}\right\rangle$ y $\left|\Psi_{0}^{\prime}\right\rangle$ representan el mismo estado inicial, pero expresado en bases diferentes.

(c) $U_{t} \mathrm{y} U_{t}^{\prime}$ representan el mismo operador de evolución, pero correlacionando bases diferentes.

Ahora bien, el operador de evolución es función del hamiltoniano de interacción $H_{\text {int }}$ entre $S$ y $M$, definido por las características físicas del aparato $M$ :

$$
U_{t}=U_{t}^{\prime}=e^{-i H_{\text {int }} t}
$$

Dicho hamiltoniano es el que introduce la correlación entre los autoestados del observable $A$ del sistema a medir y los autoestados del puntero $P$ del aparato. Para ello, $H_{\text {int }}$ debe tener una forma muy precisa, que depende de $A$ y de $P$ (cf. Mittelstaedt, 1998):

$$
H_{\text {int }}=-\frac{\lambda \hbar}{\Delta t}\left(A \otimes R^{P}\right)
$$


donde $\lambda$ es una constante, $\Delta t$ es el intervalo que dura la interacción y $R^{P}$ es el observable canónicamente conjugado del puntero $P,\left[P, R^{P}\right]=$ ith. Si, bajo el supuesto de nuestro argumento, $U_{t}$ y $U_{t}$ ' expresaran el mismo operador de evolución y éste pudiera correlacionar bases diferentes, entonces el hamiltoniano de interacción $H_{\text {int }}$ debería también correlacionar los autoestados del observable $A^{\prime}$ del sistema a medir y los autoestados del puntero $P^{\prime}$ y, por lo tanto, debería también poder expresarse como

$$
H_{\text {int }}=-\frac{\lambda \hbar}{\Delta t}\left(A^{\prime} \otimes R^{P^{\prime}}\right)
$$

donde, por hipótesis,

$$
A^{\prime} \neq A \quad \text { y } \quad P^{\prime} \neq P
$$

Por lo tanto, de las ecuaciones (6.5) y (6.6) debería cumplirse que

$$
A \otimes R^{P}=A^{\prime} \otimes R^{P^{\prime}}
$$

Pero el cálculo matricial nos enseña que, dadas las matrices $X$ e $Y$ y $X^{\prime}$ e $Y^{\prime}$,

$$
\text { Si } X \otimes Y=X^{\prime} \otimes Y^{\prime} \text {, entonces } X=X^{\prime} \text { e } Y=Y^{\prime}
$$

Por lo tanto, la única manera de que se cumpla la igualdad de la ecuación (6.8) es que

$$
A=A^{\prime} \text { y } P=P^{\prime}
$$

De este modo, hemos llegado a una contradicción entre las ecuaciones (6.7) y (6.10), lo cual indica que las premisas de partida del argumento, (a), (b) y (c), no pueden ser todas ellas verdaderas. Y si aceptamos la premisa (a) por ser el supuesto básico del argumento de Zurek, entonces las premisas (b) y (c) no pueden ser ambas verdaderas, y ello significa que, en ningún caso, las expresiones (6.1) y (6.2) pueden describir la misma medición expresada en bases diferentes.

En, definitiva, este argumento por el absurdo nos muestra que el observable que se mide no queda determinado por la base en la que se expresa el estado final de la medición, sino por el proceso que, partiendo del estado inicial, establece las correlaciones. Con independencia de cuál sea ese estado inicial, dicho proceso queda unívocamente definido por el operador de evolución $U_{t}=e^{-i H_{\text {int }} t}$ que viene fijado por la construcción del aparato de medición. En otras palabras, es el aparato de medición el que fija la base privilegiada en la medida en que su $H_{\text {int }}$ correlaciona $A$ y $P$, y no cualquier otro par $A^{\prime}$ y $P^{\prime}$ 'diferente del primero. 
Ciertamente, este resultado no debería sorprendernos. En efecto, el hecho de que la base privilegiada quede determinada por la forma del hamiltoniano de interacción no es más que una formulación teórica general de un hecho empírico bien conocido por cualquier físico experimental: la base que define los coeficientes del estado a medir viene determinada por la construcción del aparato de medición. Por ejemplo, en el caso de la medición tipo Stern-Gerlach, si en lugar de colocar los detectores en dirección $z$ ("arriba-abajo"), se los coloca en dirección $x$ ("derecha-izquierda"), con el propósito de medir el spin $S_{x}$ en dirección $x$, el resultado de esta "medición" será que no habrá detección alguna. Y esto se debe a que el imán genera un campo magnético en dirección $z$ y no en dirección $x$ : el aparato ha sido diseñado de modo tal que el momento $P_{z}$ cumpla el papel de puntero. Si en lugar de medir el estado en la base del spin $S_{z}$ deseamos medirlo en la base del spin $S_{x}$, debemos rotar el imán $90^{\circ}$ de modo tal que el nuevo campo magnético correlacione $S_{x} \operatorname{con} P_{x}$ en lugar de $S_{z} \operatorname{con} P_{z}$. En términos precisos y generales, si en un proceso de medición cuántica deseamos medir los coeficientes $c^{\prime}{ }_{i}$ del estado expresado en la base del observable $A$ ' mediante el puntero $P$ ', en lugar de los coeficientes $c i$ de $\left|\Psi_{s}\right\rangle$ expresado en la base de $A$ mediante el puntero $P$, debemos cambiar el aparato de medición, esto es, reemplazar el aparato original con su hamiltoniano de interacción $H_{\text {int }}=f(A, P)$ por un nuevo aparato con $\mathrm{H}_{\text {int }}=f\left(A^{\prime}, P^{\prime}\right)$. $\mathrm{Y}$, ciertamente, el entorno no ha jugado papel alguno en la selección de la base privilegiada determinada por $A$ y $P$.

\section{Conclusiones}

Durante las últimas décadas se ha producido una explosión en los estudios sobre decoherencia en el ámbito de la física. En particular, la investigación de este fenómeno ha cobrado una especial importancia en la llamada "computación cuántica", donde la decoherencia constituye el mayor obstáculo al que debe enfrentarse la implementación de sistemas de información que aprovechan las superposiciones cuánticas. Sin embargo, las discusiones teóricas en torno a los fundamentos de la teoría continúan presentando algunos ángulos oscuros.

En el presente trabajo hemos analizado el problema de la base privilegiada, un problema que comienza a admitirse como tal desde el mismo momento en que la teoría de decoherencia se propone como su solución. Mediante diferentes argumentos hemos sostenido que, en realidad, se trata de un pseudo-problema, construido ad hoc sobre la base de una incorrecta concepción de la medición cuántica. Cuando, por el contrario, la medición cuántica se concibe como un proceso que involucra el estado inicial del sistema a medir y el arreglo experimental mediante el cual se efectúa la me- 
dición, el problema de la base privilegiada se disuelve: no existe ambigüedad alguna respecto de la base de medición y, por tanto, la decoherencia inducida por el entorno no resulta necesaria para la selección de dicha base. Los físicos experimentales pueden continuar efectuando sus mediciones cuánticas sin preocuparse por el problema de la base privilegiada, tal como de hecho han venido haciéndolo desde hace casi un siglo.

Agradecimientos. Agradecemos a los árbitros anónimos por sus atentas lecturas del trabajo y sus oportunas sugerencias. El presente trabajo ha sido posible gracias a subsidios otorgados por la Agencia de Promoción Científica y Tecnológica (ANCyT), el Consejo Nacional de Investigaciones Científicas y Técnicas (CONICET), la Universidad de Buenos Aires (UBA) y la Sociedad Argentina de Análisis Filosófico (SADAF).

Olimpia LomBARDI Investigadora del Consejo Nacional de Investigaciones Científicas y Tecnológicas, Universidad de Buenos Aires, Argentina. olimpiafilo@arnet.com.ar Leonardo VANN I Profesor del Instituto AFE, Universidad de Buenos Aires, Argentina. lv@iafe.uba.ar

\begin{abstract}
The problem of the preferred basis consists in accounting for the basis that defines the measured observable in a quantum measurement, given the supposed theoretical ambiguity in the definition of such an observable. The purpose of this paper is to analyze this scarcely explored problem. The final aim consists in arguing that, contrary to a widespread opinion, the theory of decoherence does not supply a solution to this problem, not due to its own inability, but because the problem is actually a pseudo-problem which does not require a solution.
\end{abstract}

KEYwORDS $・$ Measuring apparatus. Environment. Basis of the Hilbert space. Superposition.

\title{
REFERENGIAS BIBLIOGRÁFIGAS
}

Adler, S. Why decoherence has not solved the measurement problem: a response to P. W. Anderson. Studies in History and Philosophy of Modern Physics, 34, B, p. 135-4,2, 2003.

Auletta, G. Foundations and interpretation of quantum mechanics. Singapore: World Scientific, 2000. Ballentine, L. Quantum mechanics: a modern development. Singapore: World Scientific, 1998. 
Medición GUÁntiGa y deGoHerencia: ¿QUé Medimos GUANdo medimos?

Blanchard, P.; Giulini, D.; Joos, E.; Kiefer, G. \& Stamatescu, I. O. (Ed.). Decoherence: theoretical, experimental, and conceptual problems. Heidelberg/Berlin: Springer, 2000. (Lecture Notes in Physics, $5^{38}$ ).

Buв, J. Interpreting the quantum world. Cambridge: Cambridge University Press, 1997.

Castagnino, M. \& Lombardi, O. The role of the Hamiltonian in the interpretation of quantum mechanics. Journal of Physics. Conferences Series, 28, \#, p. 012014, 2008.

D’Espagnat, B. A note on measurement. Physics Letters a, 282, p. 133-7, 2000.

Elby, A. The "decoherence". Approach to the measurement problem in quantum mechanics. Proceedings of the 1994 Biennial Meeting of the Philosophy of Science Association, Vol. 1. East Lansing: Philosophy of Science Association, p. 355-65. 1994, v. 1.

Heisenberg, W. Über den anschaulichen Inhalt der quantentheoretischer Kinematic und Mechanik. Zeitschrift für Physik, 43, p. 172-98, 1927.

HeIss, D. (Ed.). Fundamentals of quantum information: quantum computation, communication, decoherence and all that. Heidelberg-Berlin: Springer, 2002.

Hiley, B. J. \& PeAt, F. D. (Ed.). Quantum implications. London: Routledge and Kegan Paul, 1987.

Hughes, R. I. G. The structure and interpretation of quantum mechanics, Cambridge: Harvard University Press, 1989.

Jammer, M. The philosophy of quantum mechanics. New York: John Wiley \& Sons, 1974.

Joos, E. Elements of environmental decoherence. In: Blanchard, P.; Giulini, D.; Joos, E.; Kiefer, C. \& Stamatescu, I. O. (Ed.). Decoherence: theoretical, experimental, and conceptual problems. Heidelberg/ Berlin: Springer, 2000. p. 1-17. (Lecture Notes in Physics, 538).

Leggett, A. J. Reflections on the quantum measurement paradox. In: Hiley, B. J. \& Peat, F. D. (Ed.). Quantum implications. London: Routledge and Kegan Paul, 1987.p. 85-104.

Lombardi, O. \& Castagnino, M. A modal-hamiltonian interpretation of quantum mechanics. Studies in History and Philosophy of Modern Physics, 39, p. 380-44,3, 2008.

Mittelstaedt, P. The interpretation of quantum mechanics and the measurement process. Cambridge: Cambridge University Press, 1998.

PAZ, J. P. \& ZuREK, W. H. Environment-induced decoherence and the transition from quantum to classical. In: Heiss, D. (Ed.). Fundamentals of quantum information: quantum computation, communication, decoherence and all that. Heidelberg/Berlin: Springer, 2002. p. 77-14,8.

Pessoa Júnior, O. Conceitos de física quântica. São Paulo: Editora Livraria da Física, 2003.

Schlosshauer, M. Decoherence, the measurement problem, and interpretations of quantum mechanics. Reviews of Modern Physics, 76, p. 1267-305, 2004.

Decoherence and the quantum-to-classical transition. Berlin/Heidelberg: Springer/Verlag, 2007.

Wallace, D. Worlds in Everett interpretation. Studies in History and Philosophy of Modern Physics, 33, p. $637-61,2002$.

. Everett and structure. Studies in History and Philosophy of Modern Physics, 34, p. 87-105, 2003.

ZEH, D. On the interpretation of measurement in quantum theory. Foundations of Physics, 1, p. 69-76, 1970.

ZureK, W. H. Pointer basis of quantum apparatus: into what mixture does the wave packet collapse? Physical Review d, 24, p. 1516-25, 1981.

. Environment-induced superselection rules. Physical Review d, 26, p. 1862-80, 1982.

Decoherence and the transition from quantum to classical. Physics Today, 44, p. 36-44, 1991.

Decoherence, einselection, and the quantum origins of the classical. Reviews of Modern Physics, 75 , p. $715^{-76,2003 .}$ 